\title{
BMJ Open Role of probiotics VSL\#3 in prevention of suspected sepsis in low birthweight infants in India: a randomised controlled trial
}

Anju Sinha, ${ }^{1}$ Subodh S Gupta, ${ }^{2}$ Harish Chellani, ${ }^{3}$ Chetna Maliye, ${ }^{2}$ Vidya Kumari, ${ }^{3}$ Sugandha Arya, ${ }^{3}$ BS Garg, ${ }^{2}$ Sunita Dixit Gaur, ${ }^{4}$ Rajni Gaind, ${ }^{3}$ Vijayshri Deotale, ${ }^{2}$ Manish Taywade, ${ }^{2}$ MS Prasad, ${ }^{3}$ Vasantha Thavraj, ${ }^{1}$ Ajit Mukherjee, ${ }^{1}$ Malabika Roy ${ }^{1}$

To cite: Sinha A, Gupta SS, Chellani $\mathrm{H}$, et al. Role of probiotics VSL\#3 in prevention of suspected sepsis in low birthweight infants in India: a randomised controlled trial. BMJ Open 2015;5:e006564. doi:10.1136/bmjopen-2014006564

- Prepublication history and additional material is available. To view please visit the journal (http://dx.doi.org/ 10.1136/bmjopen-2014006564).

Received 12 September 2014 Revised 22 May 2015 Accepted 13 June 2015

CrossMark

\begin{abstract}
${ }^{1}$ Department of Reproductive and Child Health, Indian Council of Medical Research, New Delhi, Delhi, India ${ }^{2}$ Mahatma Gandhi Institute of Medical Sciences, Sewagram, Maharashtra, India

${ }^{3}$ Safdarjung Hospital, New

Delhi, Delhi, India

${ }^{4}$ District Hospital Wardha,

Wardha, Maharashtra, India
\end{abstract}

Correspondence to

Dr Anju Sinha;

apradhandr@gmail.com

\section{ABSTRACT}

Objectives: To assess the effect of the probiotic VSL\#3 in prevention of neonatal sepsis in low birthweight (LBW) infants.

Design: Randomised, double-blind, placebo-controlled trial.

Setting: Community setting in rural India.

Participants: LBW infants aged 3-7 days.

Interventions: Infants were randomised to receive probiotic (VSL\#3, 10 billion colony-forming units (cfu)) or placebo for 30 days, and were followed up for 2 months.

Main outcome measure: Possible serious bacterial infection (PSBI) as per the Integrated Management of Neonatal Childhood Illnesses algorithm, as diagnosed by fieldworkers/physicians.

Results: 668 infants were randomised to VSL\#3 and 672 to placebo. By intention-to-treat analysis, the risk of PSBI among infants in the overall population of LBW infants was not statistically significant (RR 0.79 $(95 \% \mathrm{Cl} 0.56$ to 1.03$))$. Probiotics reduced median days of hospitalisation ( 6 days vs 3 days in probiotics) $(p=0.018)$ but not the risk of hospitalisation (RR 0.66 ( $95 \% \mathrm{Cl} 0.42$ to 1.04 ). The onset of PSBI in $10 \%$ of infants occurred on the 40th day in the probiotics arm versus the 25th day in the control arm ( $p=0.063)$.

Conclusions: Daily supplementation of LBW infants with probiotics VSL\#3 (10 billion cfu) for 30 days led to a non-significant $21 \%$ reduction in risk of neonatal sepsis. A larger study with sufficient power and a more specific primary end point is warranted to confirm the preventive effect of VSL\#3 on neonatal sepsis in LBW infants.

Trial registration number: The study is registered at the Clinical Trial Registry of India (CTRI/2008/091/ 000049).

\section{INTRODUCTION}

Neonatal infections are responsible for more than a quarter of the 1 million neonatal deaths every year in India. ${ }^{1}$ Low birthweight

\section{Strengths and limitations of this study}

- Low birthweight (LBW) neonates are at high risk for infections, including neonatal sepsis.

- Probiotics are effective in preventing neonatal necrotising enterocolitis and nosocomial infections in preterm LBW babies.

- In our study, daily supplementation of LBW infants with probiotics VSL\#3 (10 billion colonyforming units (cfu)) for 30 days led to a nonsignificant $21 \%$ reduction in risk of neonatal sepsis. A significant effect was observed among infants weighing $1.5-1.9 \mathrm{~kg}$. Survival analysis showed a 15 day delay in the onset of sepsis in the intervention arm.

- Our study used the Integrated Management of Neonatal and Childhood IIInesses (IMNCI) algorithm for diagnosis of possible serious bacterial infection (PSBI) (PSBI-suspected sepsis) by fieldworkers. A larger study with sufficient power and a more specific primary end point (such as physician's diagnosis of neonatal sepsis) is warranted to confirm the preventive effect of VSL\#3 on neonatal sepsis in LBW infants.

- Our study was not powered to assess the role of probiotics on neonatal mortality. The enrolments were made during 3-7 days of life, and therefore the role of probiotics on early onset sepsis could not be evaluated.

(LBW) is a very important indirect cause of death in neonates, accounting for $40-80 \%$ of neonatal deaths. ${ }^{2}$ Infections (sepsis, pneumonia and meningitis) are known to evolve more rapidly in LBW infants, leading to severely increased disease and higher rate of death. Prevention of infection in LBW babies would directly decrease neonatal morbidity and mortality. Management of neonatal sepsis with antibiotics faces the problem of drug resistance, attributed to availability over the counter, indiscriminate use and 
incomplete courses in India. Researchers are evaluating immunotherapy (with immune globulin, myeloid colony stimulating factors, probiotics, glutamine supplementation, recombinant human protein $\mathrm{C}$ and lactoferrin) as adjuvants for the prevention of neonatal sepsis. ${ }^{3}$

Probiotics have attracted much interest and debate in the neonatal literature during the past decade. ${ }^{4}$ The FAO/WHO defines probiotics as live microorganisms that, when administered in adequate amounts, confer a health benefit on the host. ${ }^{5}$ Probiotic microorganisms have particular characteristics: human origin, safety in human use, bile acid resistance, survival in the intestine, temporary colonisation of the gut, adhesion to the mucosa and bacteriocine production. The ingestion of probiotics is associated with modification in physiological homeostasis of the intestinal flora, which is important in preventing disease, especially infections. ${ }^{6}$ The best evidence for efficacy of specific probiotic strains has been obtained with randomised controlled trials and meta-analysisis in the prevention and treatment of antibiotic-associated diarrhoea, ${ }^{7}$ gastroenteritis and acute diarrhoea, ${ }^{8}$ and in the alleviation of lactose intolerance. $^{9}$

Clinical trials evaluating the role of probiotics (Infloran) in preterm very low birthweight infants ${ }^{10-12}$ reported a reduction in the incidence of necrotising enterocolitis (NEC), overall mortality ${ }^{10}$ and severity of NEC. ${ }^{11}$ A meta-analysis ${ }^{13}$ and systematic reviews ${ }^{14}{ }^{15}$ of a randomised trial suggested a beneficial effect of probiotic treatment on reducing the incidence and all-cause mortality due to NEC. Following on from the evidence on very LBW (VLBW) and premature infants, we hypothesised that the probiotic preparationVSL\#3 might reduce morbidity due to sepsis in LBW infants. We aimed to estimate reduction in the incidence of suspected sepsis in 0-2-month-old low birthweight infants in the intervention arm with a daily supplementation of probiotic VSL\#3, 10 billion colony-forming units (cfu) over a period of 30 days. If proven to be efficacious, it could be an important public health intervention for prevention of neonatal infections.

\section{METHODS}

\section{Study design and participants}

We undertook a randomised, double-blind, placebocontrolled (1:1) trial from January 2009 to November 2011 at two tertiary care hospitals and the adjoining community areas (Safdarjung hospital in New Delhi and Mahatma Gandhi Institute of Medical Sciences Wardha, India). We screened newborn infants aged 3 days, born in the hospitals weighing $1500-2500 \mathrm{~g}$, residing within 20-25 km of the hospital, and not planning to shift residence for at least the next 2 months. We excluded extremely premature infants ( $<34$ weeks), sick infants, those with congenital malformations incompatible with life, and those with guardians not giving consent and belonging to out of study areas. Eligible babies, for whom parents/guardians gave informed consent, were enrolled on days 3-7 of life. Participants were enrolled by a physician in the hospital and followed up in the community for 2 months for occurrence of neonatal sepsis and other morbidities. Baseline information on demographic characteristics was obtained for assessment of Standard of Living Index. ${ }^{16}$ Ethical clearance was obtained from the two participating institutes. A Data Safety and Monitoring Committee (DSMC) met every 6 months and reviewed severe adverse events.

\section{Study medication}

Infants were randomly assigned to receive probiotic or placebo by the study physician. The intervention consisted of administration of the probiotic preparation VSL\#3 (a mix of eight strains: Streptococcus thermophilus, Bifidobacterium breve, Bifidobacterium longum, Bifidobacterium infantis, Lactobacillus acidophilus, Lactobacillus plantarum, Lactobacillus paracasei and Lactobacillus delbrueckii spp bulgaricus, at a dose of 10 billion cfu for 30 days, starting on the third day of life. The content of the probiotic sachet was mixed in expressed breast milk in a plastic cup and fed to the infant. A sterilised plastic cup and stirrer were provided along with the sachets. A similar-looking maltodextrin preparation in the same outer packing was administered to the control group. The supplement was prepared by CD Pharma India Pvt. Ltd. The preparations withstood a temperature up to $28^{\circ} \mathrm{C}$ and were therefore kept in a cold chain (refrigerators/vaccine carriers) at the homes of enrolled infants.

\section{Randomisation and masking}

A computer generated stratified block randomisation with a permuted block size of four was used. We stratified infants by birth weight (1500-2000 g, 2001-2500 g) and sex. A team of scientists at INCLEN Trust, New Delhi, used a computer-generated table for subject allocation. Allocation concealment was ensured by sequentially numbering the sachet packets containing VSL\#3 or placebo after block randomisation. Identical packaging of VSL\#3 and a placebo with similar consistency and colour was provided. Parents of enrolled infants, investigators and fieldworkers were masked to treatment allocation. Data analysis was performed in a blinded manner. The codes remained with the INCLEN Trust, and were disclosed to the DSMB and ICMR on completion of data analysis.

\section{Follow-up and assessment}

Follow-up visits were carried out by the fieldworker for supervising supplementation over 30 days, and detection of morbidities over 2 months. Visitation was daily during the first week, biweekly in weeks $2-4$ of life, and weekly in the second month. Detection of neonatal sepsis was performed during visits, using the Integrated Management of Neonatal and Childhood Illnesses (IMNCI) algorithm (http://www.unicef.org/india/Training_Module_1-9) for detection of possible serious bacterial infection 
(PSBI) suggested by the presence of any of the following signs of infection: convulsions or fast breathing (60 breaths per minute or more); severe chest in-drawing or nasal flaring or grunting; 10 or more skin pustules or a large boil; axillary temperature $37.5^{\circ} \mathrm{C}$ or above (or feels hot to touch); temperature less than $35.4^{\circ} \mathrm{C}$ (or feels cold to touch); lethargic or unconscious or less than normal movements. Fieldworkers referred and accompanied sick infants to the study hospital for treatment. At the hospital, the infants were examined by a physician, blood cultures were obtained, and treatment was carried out as per the protocol of the hospital.

Information on compliance and morbidities was recorded. An enrolment card was provided which parents were asked to carry whenever they sought treatment for the infant in between study visits. Efforts were made to contact local practitioners visited independently by parents of infants and to collect the details of treatments prescribed. Study staff were trained in the IMNCI algorithm and given practice on eliciting signs of neonatal sepsis. Study procedures were standardised and regular exercises were conducted so as to reduce interobserver and intraobserver variability. Quality assurance measures included supervisory checks in the fieldwork, data collection and data cleaning. All case record forms were cross-checked by supervisors and medical officers before being sent for double data entry (in EPI Info V.6.0) with built-in range and consistency checks. (Details on quality assurance mechanism for the study implementation is given as web appendix).

The primary outcome was risk of PSBI as per the IMNCI algorithm, as diagnosed by fieldworkers or physicians. Secondary outcomes were estimation of the effect of VSL\#3 on overall morbidity pattern in 0-2-month-old LBW infants; stool colonisation patterns in $10 \%$ of subjects; and assessment of side effects due to the probiotic VSL\#3, if any. On the recommendation of the DSMC, data on diagnosis of sepsis by a physician was also recorded as an amendment to the protocol.

\section{Gut colonisation substudy}

Data on gut colonisation was important to substantiate the clinical findings. Stool samples from 202 (101 each in the intervention and placebo arms) enrolled infants were collected on day ' 1 ', day ' 21 ' and day ' 60 ' to correspond to the end of follow-up.

The samples were collected in sterile specimen jars (plastic containers) and transported to the lab at $4^{\circ} \mathrm{C}$, and stored at $-20^{\circ} \mathrm{C}$. Processing was completed within 10 days to evaluate their bacterial microflora composition and enzymatic activities. Sequencing and real-time PCR were conducted on DNA samples extracted from stool specimens.

\section{Statistical analysis}

Bang $e t a l^{2}$ reported a $17 \%$ incidence of neonatal sepsis in the community. Assuming a $10 \%$ loss to follow-up, 1340 infants were needed (670 in each group) to observe a $30 \%$ reduction in incidence of sepsis at $5 \%$ significance with $80 \%$ power. Analyses were performed by intention to treat. Software ' $\mathrm{R}$ '17 (V.3.0.0) was used for calculation of PSBI risk, incidence rates, CIs and incidence rate ratios. We used Kaplan-Meier survival analysis curves with Herrington Flemming variation ${ }^{18}$ of the log rank test to compare the survival curves in the probiotic and placebo arms. We used a 't test' after log conversion to compare colony counts groups in the gut colonisation substudy.

\section{Role of the funding source}

Funding source played no role in the study design, data collection, analysis and interpretation, writing of the report or decision to submit it for publication.

\section{RESULTS}

Between January 2009 and November 2011, 5927 LBW newborn infants were screened and 1340 eligible LBW infants were enrolled (figure 1). Of the 5927 screened, 4587 were excluded (reasons given in figure 1). The probiotic and placebo groups were comparable with regard to baseline characteristics such as mode of delivery, mean birth weight, mother's schooling, religion of the family, standard of living index (SLI), and maternal morbidities during current pregnancy (table 1).

The intervention and control groups were similar in the mean number of fieldworker visits performed (20.8 \pm 3.7 in probiotic vs $20.5 \pm 4.0$ in placebo groups; $\mathrm{p}=0.154)$, mean number of doses of interventional product consumed $(29.1 \pm 4.4$ in probiotics vs $28.7 \pm 5.2$ in placebo; $\mathrm{p}=0.129$ ), and mean number of days of follow-up visits $(56.3 \pm 2.2$ in probiotics vs $56.1 \pm 3.8$ in placebo; $\mathrm{p}=0.239$ ).

\section{Primary outcome: PSBI}

On the basis of the intention-to-treat (ITT) analysis, there was a non-significant $21 \%$ reduction in the overall risk of PSBI in the probiotic group (84 cases in 688 infants in the probiotic arm versus 107 cases in 672 infants in the placebo arm; RR 0.79 (95\% CI 0.56 to 1.03 ); $\mathrm{p}=0.080$ ) (table 2). In the probiotic group, there was a significant $71 \%$ reduction in risk in the un prespecified subgroup of infants with birth weights $1.5-1.99 \mathrm{~kg}$ (4 cases in 74 infants in probiotics vs 14 cases in 75 infants in the placebo arm; RR 0.29 (95\% CI 0.10 to $0.84) ; \mathrm{p}=0.014)$. A $32 \%$ reduction in the risk of PSBI among the un prespecified subgroup of female infants was observed (36 cases in 348 infants in probiotic vs 53 cases in 349 infants in the placebo group; RR 0.68 (95\% CI 0.46 to 0.99 ); $\mathrm{p}=0.056$ ). There was no evidence of an interaction effect in the un prespecified subgroup analysis ( $p$ value $=0.128$ for the interaction term between treatment and birthweight group).

We also calculated the incidence rates of PSBI computed with the person-time data collected during home visits (table 3). The PSBI incidence rate in the probiotics arm was 2.61/1000 days of follow-up versus 3.40/ 
Figure 1 Participant flow through the trial.

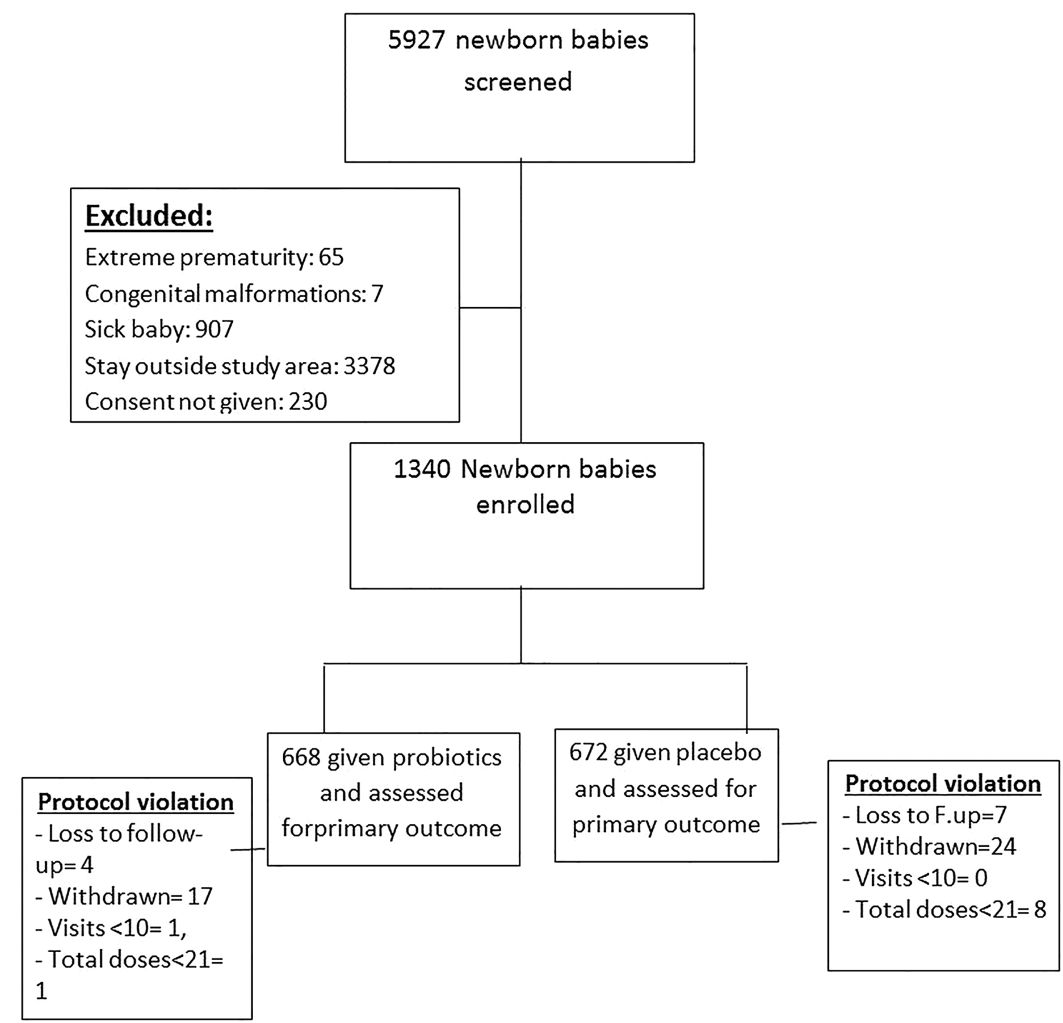

\begin{tabular}{|c|c|c|c|c|}
\hline & \multicolumn{2}{|c|}{$\begin{array}{l}\text { Probiotics } \\
(\mathrm{N}=668)\end{array}$} & \multicolumn{2}{|c|}{$\begin{array}{l}\text { Placebo } \\
(\mathrm{N}=672)\end{array}$} \\
\hline \multicolumn{5}{|l|}{ Sex } \\
\hline Male & 319 & $47.8 \%$ & 320 & $47.6 \%$ \\
\hline Female & 349 & $52.2 \%$ & 352 & $52.4 \%$ \\
\hline \multicolumn{5}{|l|}{ Birthweight groups } \\
\hline $1500-1999 \mathrm{~g}$ & 74 & $11.1 \%$ & 75 & $11.2 \%$ \\
\hline $2000-2499 \mathrm{~g}$ & 594 & $88.9 \%$ & 597 & $88.8 \%$ \\
\hline Mean (SD) birth weight & \multicolumn{2}{|c|}{$2261 \pm 179$} & \multicolumn{2}{|c|}{$2263 \pm 179$} \\
\hline \multicolumn{5}{|c|}{ Mother's schooling (years) } \\
\hline$\leq 8$ & 292 & $43.7 \%$ & 285 & $42.4 \%$ \\
\hline$>8$ & 376 & $56.3 \%$ & 387 & $57.6 \%$ \\
\hline \multicolumn{5}{|l|}{ Religion } \\
\hline Hindu & 489 & $73.2 \%$ & 501 & $74.6 \%$ \\
\hline Muslim & 46 & $6.9 \%$ & 41 & $6.1 \%$ \\
\hline Others & 133 & $19.9 \%$ & 130 & $19.3 \%$ \\
\hline \multicolumn{5}{|l|}{ Standard of living index } \\
\hline Low & 98 & $14.7 \%$ & 85 & $12.6 \%$ \\
\hline Medium & 348 & $52.1 \%$ & 382 & $56.8 \%$ \\
\hline High & 222 & $33.2 \%$ & 205 & $30.5 \%$ \\
\hline \multicolumn{5}{|l|}{ Mode of delivery } \\
\hline Vaginal & 633 & $94.8 \%$ & 629 & $93.6 \%$ \\
\hline LSCS+others & 35 & $5.2 \%$ & 43 & $6.4 \%$ \\
\hline \multicolumn{5}{|c|}{ Morbidities during pregnancy } \\
\hline Hypertension & 23 & $3.4 \%$ & 18 & $2.7 \%$ \\
\hline Anaemia & 55 & $8.2 \%$ & 63 & $9.4 \%$ \\
\hline PROM & 22 & $3.3 \%$ & 30 & $4.5 \%$ \\
\hline None & & $85.0 \%$ & 561 & $83.5 \%$ \\
\hline Mean SLI score & \multicolumn{2}{|c|}{$22.2 \pm 7.9$} & \multicolumn{2}{|c|}{$22.3 \pm 7.7$} \\
\hline
\end{tabular}

1000 days in the placebo arm (RR 0.77 (95\% CI 0.59 to 0.99), $\mathrm{p}=0.0493$ ). Among the un prespecified subgroup of babies weighing $1.50-1.99 \mathrm{~kg}$, the incidence rate of PSBI/1000 days was 1.67 and 4.57 in the probiotic and placebo groups, respectively (RR 0.36 (95\% CI 0.15 to $0.87 ; \mathrm{p}=0.008)$.

\section{Secondary outcomes \\ Other morbidities}

There was no significant difference between the groups for the proportion of babies who had local infection (3.0\%; (95\% CI $2.0 \%$ to $4.7 \%$ ) in probiotic vs $3.4 \%$; (95\% CI $2.2 \%$ to $5.0 \%)$ in the placebo group, $\mathrm{p}=0.69)$, feeding problems $(18.9 \%$; (95\% CI $16.0 \%$ to $22.0 \%)$ in probiotic vs $16.4 \%$; (95\% CI $13.7 \%$ to $19.3 \%$ ) in the placebo group, $\mathrm{p}=0.21)$, or other morbidities $(35.9 \%$; (95\% CI $32.4 \%$ to $39.6 \%)$ in probiotic vs $34.2 \%$; (95\% CI $30.7 \%$ to $37.9 \%$ ) in the placebo group, $\mathrm{p}=0.52$ ).

\section{Gut colonisation}

There were differences in absolute colony counts in the two groups on days 1, 21 and 60; however, these differences were not significant statistically.

The difference between colony counts in the probiotic and placebo groups (day 21-day 1) was statistically significant $(p=0.0476)$ for $L$. acidophilus; however, it was not significant for $S$. thermophilus $(\mathrm{p}=0.9964)$ and B. longum ( $\mathrm{p}=0.3872)$. Colonisation was also observed in the placebo arm, most likely due to exclusive breastfeeding. 
Table 2 Cumulative risk of PSBI/clinically suspected sepsis

\begin{tabular}{|c|c|c|c|c|c|c|c|c|c|c|c|}
\hline & \multicolumn{4}{|c|}{ Probiotics } & \multicolumn{4}{|c|}{ Placebo } & \multicolumn{2}{|c|}{$\begin{array}{l}\text { Cumulative } \\
\text { risk ratio }\end{array}$} & \multirow[b]{3}{*}{ p Value* } \\
\hline & \multirow[b]{2}{*}{$\mathbf{N}$} & \multirow[b]{2}{*}{$\mathbf{N}$} & \multicolumn{2}{|c|}{ Cumulative risk } & \multirow[b]{2}{*}{$\mathbf{N}$} & \multirow[b]{2}{*}{$\mathbf{N}$} & \multicolumn{2}{|c|}{ Cumulative risk } & \multirow[b]{2}{*}{$\mathbf{R R}$} & \multirow[b]{2}{*}{$95 \% \mathrm{Cl}$} & \\
\hline & & & (\%) & $95 \% \mathrm{CI}$ & & & $(\%)$ & $95 \% \mathrm{Cl}$ & & & \\
\hline \multicolumn{12}{|c|}{ (PSBI, by field investigator) } \\
\hline All strata & 84 & 668 & 12.6 & 10.3 to 15.3 & 107 & 672 & 15.9 & 13.3 to 18.9 & 0.79 & 0.56 to 1.03 & 0.080 \\
\hline $1.5-1.99 \mathrm{~kg}$ & 4 & 74 & 5.4 & 1.7 to 13.49 & 14 & 75 & 18.7 & 11.3 to 29.1 & 0.29 & 0.10 to 0.84 & 0.014 \\
\hline $2.0-2.49 \mathrm{~kg}$ & 80 & 594 & 13.5 & 11.0 to 16.5 & 93 & 597 & 15.6 & 12.9 to 18.7 & 0.86 & 0.66 to 1.14 & 0.303 \\
\hline Male & 48 & 320 & 15.0 & 11.5 to 19.4 & 54 & 323 & 16.7 & 13.0 to 21.2 & 0.90 & 0.63 to 1.28 & 0.553 \\
\hline Female & 36 & 348 & 10.3 & 7.5 to 14.0 & 53 & 349 & 15.2 & 11.8 to 19.4 & 0.68 & 0.46 to 0.99 & 0.056 \\
\hline \multicolumn{12}{|c|}{ Suspected sepsis (by physician) } \\
\hline All strata & 38 & 668 & 5.7 & 4.2 to 7.7 & 54 & 672 & 8.0 & 6.2 to 7.7 & 0.71 & 0.47 to 1.06 & 0.091 \\
\hline $1.5-1.99 \mathrm{~kg} \dagger$ & 0 & 74 & 0.0 & 0 to 5.9 & 8 & 75 & 10.7 & 5.2 to 19.9 & - & - & 0.007 \\
\hline $2.0-2.49 \mathrm{~kg}$ & 38 & 594 & 6.4 & 4.7 to 8.7 & 46 & 597 & 7.7 & 5.8 to 10.1 & 0.83 & 0.55 to 1.26 & 0.381 \\
\hline Male & 21 & 320 & 6.6 & 4.3 to 9.9 & 30 & 323 & 9.3 & 6.6 to 13.0 & 0.71 & 0.41 to 1.21 & 0.205 \\
\hline Female & 17 & 348 & 4.9 & 3.0 to 7.7 & 24 & 349 & 6.9 & 4.6 to 10.1 & 0.71 & 0.39 to 1.30 & 0.270 \\
\hline
\end{tabular}

\section{Post hoc analyses}

A post hoc analysis based on the ITT showed a nonsignificant $29 \%$ reduction in the overall risk of physiciandiagnosed sepsis in the probiotic group (38 cases in 688 infants in the probiotic vs54 cases in 672 infants in the placebo group; RR 0.71 (95\% CI 0.47 to 1.06 ), $\mathrm{p}=0.091$ ). There was no case of suspected sepsis diagnosed by the physician in the group of 74 infants taking probiotics and weighing $1.50-1.99 \mathrm{~kg}$, as compared to eight cases in 75 infants of this weight in the placebo group (RR and $95 \%$ CI not calculated due to no sepsis cases in the probiotics group, Fisher's Exact test $\mathrm{p}$ value=0.007).
There was no evidence of an interaction effect in the un prespecified subgroup analysis ( $p$ value $=0.974$ for the interaction term between treatment and birthweight group).

In the post hoc analysis of physician-diagnosed sepsis, the incidence rate in the probiotic arm was 1.07/ 1000 days vs $1.59 / 1000$ days with placebo (RR 0.67 ; (95\% CI 0.45 to 0.99$), \mathrm{p}=0.048)$. In the $1.5-1.99 \mathrm{~kg}$ weight stratum, there was no case of sepsis diagnosed by the physician versus an incidence rate of $2.40 / 1000$ follow-up days in the placebo arm (RR $0.00(95 \%$ CI 0.0 to 0.35$) ; \mathrm{p}=0.002$ ).

Table 3 Incidence rate for PSBI clinically suspected sepsis per 1000 days of follow-up

\begin{tabular}{|c|c|c|c|c|c|c|c|c|c|c|c|}
\hline & \multicolumn{4}{|c|}{ Probiotics } & \multicolumn{4}{|c|}{ Placebo } & \multicolumn{2}{|c|}{$\begin{array}{l}\text { Incidence } \\
\text { rate ratio }\end{array}$} & \multirow[b]{3}{*}{ p Value* } \\
\hline & \multirow[b]{2}{*}{$\mathbf{n}$} & \multirow{2}{*}{$\begin{array}{l}\text { Person- } \\
\text { days }\end{array}$} & \multicolumn{2}{|c|}{$\begin{array}{l}\text { Incidence rate/ } \\
1000 \text { days }\end{array}$} & \multirow[b]{2}{*}{$\mathbf{N}$} & \multirow{2}{*}{$\begin{array}{l}\text { Person- } \\
\text { days }\end{array}$} & \multicolumn{2}{|c|}{$\begin{array}{l}\text { Incidence rate/ } \\
1000 \text { days }\end{array}$} & \multirow[b]{2}{*}{ RR } & \multirow[b]{2}{*}{$95 \% \mathrm{Cl}$} & \\
\hline & & & Rate & $95 \% \mathrm{Cl}$ & & & Rate & $95 \% \mathrm{Cl}$ & & & \\
\hline \multicolumn{12}{|c|}{ PSBIs (by field investigator) and sepsis by physician } \\
\hline All strata & 98 & 37532 & 2.61 & 2.12 to 3.18 & 128 & 37681 & 3.40 & 2.83 to 4.04 & 0.77 & 0.59 to 0.99 & 0.049 \\
\hline $1.5-1.99 \mathrm{~kg}$ & 6 & 4204 & 1.67 & 0.52 to 3.11 & 19 & 4159 & 4.57 & 2.75 to 7.13 & 0.36 & 0.15 to 0.87 & 0.008 \\
\hline $2.0-2.49 \mathrm{~kg}$ & 92 & 33328 & 2.19 & 2.23 to 3.39 & 109 & 33522 & 3.25 & 2.67 to 3.92 & 0.67 & 0.64 to 1.12 & 0.248 \\
\hline Male & 58 & 17946 & 3.23 & 2.45 to 4.18 & 69 & 18107 & 3.81 & 2.97 to 4.8 & 0.85 & 0.60 to 1.20 & 0.357 \\
\hline Female & 40 & 19586 & 2.04 & 1.46 to 2.78 & 59 & 19574 & 3.01 & 2.29 to 3.89 & 0.68 & 0.45 to 1.01 & 0.056 \\
\hline \multicolumn{12}{|c|}{ Suspected sepsis by physician } \\
\hline All strata & 40 & 37532 & 1.07 & 0.76 to 1.45 & 60 & 37681 & 1.59 & 1.21 to 2.05 & 0.67 & 0.45 to 0.99 & 0.048 \\
\hline $1.5-1.99 \mathrm{~kg} \dagger$ & 0 & 4204 & 0.00 & 0.00 to 1.11 & 10 & 4159 & 2.40 & 1.15 to 4.42 & 0.00 & 0.0 to 0.35 & 0.002 \\
\hline $2.0-2.49 \mathrm{~kg}$ & 40 & 33328 & 1.20 & 0.86 to 1.63 & 50 & 33522 & 1.49 & 1.11 to 1.97 & 0.80 & 0.53 to 1.22 & 0.307 \\
\hline Male & 23 & 17946 & 1.28 & 0.81 to 1.92 & 35 & 18107 & 1.93 & 1.35 to 2.69 & 0.66 & 0.39 to 1.12 & 0.126 \\
\hline Female & 17 & 19586 & 0.87 & 0.51 to 1.39 & 25 & 19574 & 1.28 & 0.83 to 1.89 & 0.68 & 0.37 to 1.26 & 0.221 \\
\hline
\end{tabular}

${ }^{*} p$ Values less than 0.05 have been shown in bold.

$\dagger$ As there was no case among the exposed, the risk ratio and its $\mathrm{Cl}$ were calculated by adding 0.5 to each cell. Fisher's exact $p$ value was calculated instead of $\chi^{2}$ test.

$\mathrm{PSBI}$, possible serious bacterial infections. 


\section{Comparison of event rates}

The Kaplan Meier survival analysis curves were plotted to compare the event rates in the probiotic and placebo arms (figure 2). This shows a divergence between the curves for probiotic and placebo, starting after a week of supplementation and remaining throughout the follow-up period. The onset of first episode of PSBI in $10 \%$ of infants occurred on the 41 st day in the probiotic arm versus the 24th day in the control arm $(p=0.063)$, and the onset of first episode of suspected sepsis diagnosed by the physician in $5 \%$ of infants occurred on the 53rd day in the probiotic arm versus the 26th day in the control arm $(\mathrm{p}=0.071)$.

\section{Adverse outcomes: hospitalisations and deaths}

Hospitalisation and death in enrolled infants were considered as moderate and severe adverse outcomes, respectively (table 4). During the study, 29 infants in the probiotic arm and 44 in the placebo arm needed to be hospitalised $(\mathrm{p}=0.075)$. The median number of hospitalisations was 3 days in the probiotic arm versus 6 days in the placebo arm $(p<0.018)$. There were three deaths, one in the probiotic arm and two in the placebo arm. Verbal autopsy reports of deaths reviewed by the DSMB did not attribute them to the intervention. No side effects of VSL\#3 were reported.

\section{DISCUSSION}

Overall, supplementation with the probiotic VSL\#3 in LBW infants was associated with a $21 \%$ (non-significant) reduction in the risk of suspected sepsis (PSBI) diagnosed by the fieldworker. However, in the un-prespecified subgroup of infants weighing $1.5-1.99 \mathrm{~kg}$, the reduction
Figure 2 Kaplan-Meier curves for difference between event rates in the probiotic and placebo groups. PSBI, possible serious bacterial infection.
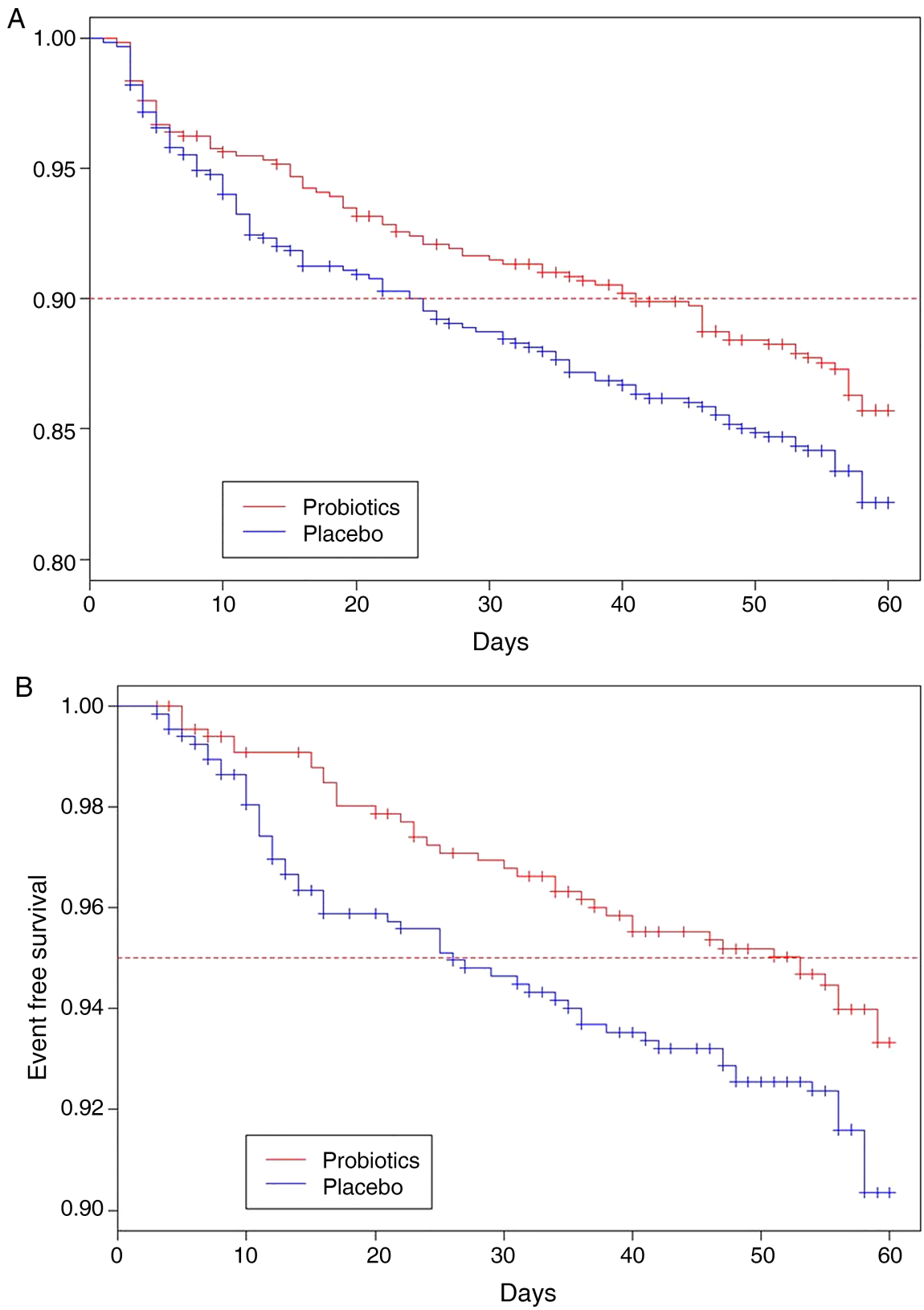
Table 4 Comparison of adverse outcomes between the probiotics and placebo arms

\begin{tabular}{|c|c|c|c|c|}
\hline & Probiotics & Placebo & Total & p Value \\
\hline Hospitalisation required & 29 & 44 & 73 & 0.075 \\
\hline \multicolumn{5}{|l|}{ Duration of hospitalisation } \\
\hline 25th centile & 2 days & 3 days & 73 & $<0.018 \dagger$ \\
\hline Median & 3 days & 6 days & & \\
\hline 75th centile & 5 days & 8.75 days & & \\
\hline Deaths & 1 & 2 & 3 & NS \\
\hline
\end{tabular}

in risk of PSBI was statistically significant (reduction of $71 \% ; \mathrm{p}=0.014$ ). The primary analysis in this study was based on the PSBI classification by the fieldworker as per the IMNCI algorithm as an indicator of neonatal sepsis. ${ }^{19}$ The classification PSBI under IMNCI is described as sensitive but not specific for detection of neonatal sepsis. ${ }^{20}$ Prior to closure of the study, the DSMC recommended conducting post hoc analyses using the physician's diagnosis of sepsis as the outcome measure. In this analysis, there is a $29 \%$ overall reduction in risk of sepsis. However, in the un prespecified subgroup of infants weighing $1.5-1.99 \mathrm{~kg}$, there is a $100 \%$ reduction, with no cases observed in the group receiving probiotic supplementation. Our findings of probiotics efficacy among infants $1.5-1.99 \mathrm{~kg}$ may be a chance finding, generating a hypothesis that this intervention may be useful for the most vulnerable of the LBW babies. Our power calculations did not consider this a priori, and hence these findings need to be confirmed in future studies. Probiotic intervention significantly reduced the mean number of hospitalisation days. The Kaplan Meier survival analysis shows a 15-day delay in the onset of sepsis in the intervention arm; this translates to a disease-free window during the 28-day period, which is crucial for neonatal survival. Moreover, considering a higher case fatality in sepsis at early ages, this becomes even more important. Our results may not be definitive or robust enough; however, there is a consistency in them, and we do not consider this as a 'negative trial'. Although our study is not large enough, it may be misleading to interpret it as proving that there is no effect of the probiotic intervention or no difference between the study groups. More evidence needs to be generated, since an interpretation of no effect might discourage further studies. ${ }^{21}$

In the current study, a consistent difference between the intervention and control groups $\mathrm{A}$ and $\mathrm{B}$ in all the tables as well as the Kaplan Meier survival analysis curves was observed. The difference between the groups was marked in most of the tables when the physician's diagnosis of sepsis was considered. After evaluating the coded results, the DSMB even considered amendment of the original protocol to change the primary outcome variable to sepsis as diagnosed by the physician so that the study conclusively finds out the role of VSL\#3 in preventing neonatal sepsis. The opinion of the DSMB members was that physicians' diagnosis of sepsis would be widely acceptable owing to its accuracy as compared to diagnosis by field workers. Committee advised extending the study to enrol more infants for the revised primary outcome. However, a 'Technical Advisory Group' (including clinical trialists, biostatisticians, public health experts), formed on recommendations of the DSMB, suggested that this study should be closed and another study planned. In view of the fact that the trial was registered and the statistical analytical plan specified the primary outcome as PSBI, it was suggested that the findings on Physician's diagnosis be presented post hoc.

The physician's diagnosis of sepsis is more meaningful than PSBI, owing to its specificity. The reported post hoc analyses increase our confidence in the results. However, physicians used their clinical judgement for diagnosing sepsis; there was no standardised definition used, and this is a limitation of the study. Future trials should evaluate the role of VSL\#3 on incidence of sepsis with a precise definition of the outcome measure. The incidence of sepsis observed in the study was lower than the expected effect size used in determining the sample size of the study. Home visits, ${ }^{22}{ }^{23}$ health education messages about exclusive breastfeeding and hygiene, and referral by fieldworkers could improve care and care-seeking, resulting in lower morbidity and mortality and a type II error for the overall result of our study. Our study has several other limitations. It was not powered to assess the role of probiotics on neonatal mortality. The enrolments were made during 3-7 day of life, so we cannot comment on the role of probiotics on early onset sepsis. We followed infants for a period of 2 months and therefore cannot comment on the long-term effects of probiotic supplementation. There are concerns regarding heterogeneity in probiotic products. The literature suggests greater protection with double or triple probiotic strains. ${ }^{13}$ Probiotic VSL\#3 is a mix of eight strains, namely $S$. thermophilus, B. breve, B. longum, B. infantis, L. acidophilus, L. plantarum, L. paracasei and L. Delbrueckii spp bulgaricus. In a randomised placebo-controlled clinical trial in India, VSL\#3 resulted in early recovery and reduced need for oral rehydration salts in rotavirusaffected children aged 6 months to 2 years. ${ }^{24}$ 
In previous studies, probiotics have been found to prevent necrotising enterocolitis (NEC) by preventing colonisation of the gut by pathogens, promoting colonisation with beneficial organisms, improving maturity and function of the gut mucosal barrier and modulating the immune system to the advantage of the host. ${ }^{11}$ 12 A Cochrane review showed moderate to low quality evidence that oral lactoferrin with or without probiotics decreases sepsis and NEC in preterm infants. ${ }^{25}$ The mechanism for efficacy of probiotics in reducing the incidence of sepsis in VLBW infants is probably similar to that for NEC. ${ }^{26}{ }^{11}$ However, in a further study by Lin et $a l,{ }^{12}$ the effect of reduction in the incidence of sepsis was not confirmed. This study was conducted among severely ill, hospitalised VLBW infants with central line, total parenteral nutrition and prolonged use of mechanical ventilation. Probiotics exert their effects by positively influencing normal microbe-microbe and host-microbe interactions and may augment the protection afforded by commensal flora through competitive interactions, direct antagonism of pathogens and/or production of antimicrobial factors. The preventive mechanisms could fail in the face of severe conditions as in case of the study by Lin $e t a l^{12}$ Probiotics alone would not overcome the infection induced by invasive procedures. However, in the community setting such as in our study, among LBW predominantly breastfed infants, probiotics could be effective in preventing sepsis, since the primary effect of orally administered probiotics is in the gastrointestinal tract with prevention of bacterial translocation.

Neonatal infection is a high priority area of research. Research on immunotherapy ${ }^{3}$ has provided very few leads. To the best of our knowledge, at present there are no proven interventions beneficial in preventing sepsis in LBW infants, ${ }^{27}$ apart from exclusive breastfeeding and practice of hygiene. This study provides an indication that microbial interference by beneficial bacteria is helpful in decreasing neonatal morbidity. Considering a $30 \%$ prevalence of LBW in India ${ }^{28}$ and $30 \%$ mortality due to sepsis in newborns, ${ }^{1}$ even a modest decline in the incidence of sepsis due to preventive intervention with probiotics could avert thousands of neonatal deaths. When produced on a large scale, it would be a costeffective intervention for a major public health problem.

We observed a significant positive treatment effect in the subgroup of infants weighing $1.5-2.0 \mathrm{~kg}$. This mandates the conduct of a larger study with sufficient power to conclusively evaluate the role of probiotics among LBW infants in a population at high risk of mortality from sepsis. There is also a need to conduct this kind of study for all neonates to assess if probiotics could be beneficial even for children who are not LBW.

Acknowledgements The authors gratefully acknowledge the contribution of Mr N C Saxena and the Project Review Group, ICMR for approving the protocol. The authors thank the Data Safety and Monitoring Committee Chairperson Dr Sesikeran, and members Dr N K Arora, Dr R M Pandey and Dr Arvind Saili for their inputs and guidance in the scientific conduct of the trial. The authors thank all field investigators, infants and their parents for participating in the study. The authors thank Mr. Shil Henba for helping with data cleaning and management. The authors thank the Civil Surgeon, Wardha and the Medical Officers at District Hospital, Wardha; and rural hospitals at Hinganghat, Pulgaon and Arvi. The authors thank all the Medical officers, Senior Residents and nurses of the Department Of Pediatrics, Safdarjung Hospital, Delhi. The authors gratefully acknowledge Dr Manoj K Das and the team of INCLEN Trust for carrying out the randomisation procedure. The authors thank Mr Kawaldeep Chaddha, Mr. Aditya Sahu and Ms. Ambika Johar from CD Pharma India Pvt Ltd for procurement and supply of VSL\#3 and placebo for the trial.

Contributors AS conceptualised the study, prepared the protocol and drafted the report. All the authors reviewed and approved it. AS, SSG, HC and BSG were responsible for the design of the trial; AS, SSG and MSP were responsible for preparing the standard operating procedures and data collection instruments; SSG, HC, CM, VK, SA, SD, VD and MT were responsible for the implementation of the trial and clinical management of subjects; SSG designed the database and managed the data; SSG and AS were responsible for the analyses and interpretation. AS, SSG, HC and SA edited the draft manuscript. VT closely monitored study implementation. AM, MR and contributed at different stages of study implementation. All authors had full access to all of the data (including statistical reports and tables) in the study and can take responsibility for the integrity of the data and the accuracy of the data analysis; they approved the final version to be published and agree to be accountable for the accuracy and integrity of the manuscript.

Funding The funding for this study was provided by the Indian Council of Medical Research. Language editing of the manuscript was provided by Jo Whelan of Textpharm Ltd and funded by VSL Pharmaceuticals Inc.

\section{Competing interests None declared.}

\section{Patient consent Obtained.}

Ethics approval Institutional Ethics Committee of Safdarjung and MGIMS Wardha Hospital and Mahatma Gandhi Institute of Medical Sciences.

Provenance and peer review Not commissioned; externally peer reviewed.

Data sharing statement No additional data are available.

Open Access This is an Open Access article distributed in accordance with the Creative Commons Attribution Non Commercial (CC BY-NC 4.0) license, which permits others to distribute, remix, adapt, build upon this work noncommercially, and license their derivative works on different terms, provided the original work is properly cited and the use is non-commercial. See: http:// creativecommons.org/licenses/by-nc/4.0/

\section{REFERENCES}

1. Bassani DG, Kumar R, Awasthi S, et al., Million death study collaborators. Causes of neonatal and child mortality in India: a nationally representative mortality survey. Lancet 2010;376:1853-60.

2. Bang TA, Baitule SB, Reddy HM, et al. Low birth weight and preterm neonates: can they be managed at home by mother and a trained village health worker? J Perinatol 2005;25:S72-81.

3. Wolkowiez MC, Benjamin DK, Capparelli E. Immunotherapy in neonatal sepsis: advances in treatment and prophylaxis. Curr Opin Pediatr 2009;21:177-81.

4. Nair V, Soraisham AS. Probiotics and prebiotics: role in prevention of nosocomial sepsis in preterm infants. Int J Pediatrics 2013;2013:8.

5. Guidelines for the evaluation of probiotics in food: report of a joint FAO/WHO Working Group. London, Ontario, Canada: Food and Agriculture Organization of the United Nations and World Health Organization, 2002

6. Montalto M, Arancio F, Izzi D. Probiotics: history, definition, requirements and possible therapeutic applications. Ann Ital Med Int 2002;17:157-65.

7. Hempel S, Newberry SJ, Maher AR, et al. Probiotics for the prevention and treatment of antibiotic-associated diarrhea: a systematic review and meta-analysis. JAMA 2012;307:1959-69.

8. Dinleyici EC, Eren M, Ozen M, et al. Effectiveness and safety of Saccharomyces boulardii for acute infectious diarrhoea. Expert Opin Biol Ther 2012;12:395-410.

9. Martean $P$, Seksik $P$, Jian R. Probiotics and intestinal health effects: a clinical perspective. Br J Nutr 2002;88(Suppl 1):S51-7. 
10. Hoyos AB. Reduced incidence of necrotizing enterocolitis associated with enteral administration of Lactobacillus acidophilus and Bifidobacterium infantis to neonates in an intensive care unit. Int $J$ Infect Dis 1999;3:197-202.

11. Lin $\mathrm{HC}$, Su BH, Chen AC, et al. Oral probiotics reduce the incidence and severity of necrotizing enterocolitis in very low birth weight infants. Pediatrics 2005;115:1-4.

12. Lin $\mathrm{HC}$, Chyong $\mathrm{HH}$, Chen $\mathrm{HL}$, et al. Oral probiotics prevent necrotizing enterocolitis in very low birth weight preterm infants: a multicenter randomized controlled trial. Pediatrics 2008;122:693-700.

13. Schanler RJ. Probiotics and necrotizing enterocolitis in premature infants. Arch Dis Child Fetal Neonatal Ed 2006;91:F399-7.

14. Alfaleh K, Anabrees J. Probiotics for prevention of necrotizing enterocolitis in preterm infants. Cochrane Database Syst Rev 2014; (4):CD005496.

15. Deshpande G, Rao S, Patole S. Probiotics for prevention of necrotising enterocolitis in preterm neonates with very low birthweight: a systematic review of randomized controlled trials. Lancet 2007;369:1614-20.

16. International Institute for Population Sciences (IIPS) and Macro International. National family health survey (NFHS-3), 2005-06. Vol I. Mumbai, India: IIPS, 2007.

17. R Development Core Team. R: language and environment for statistical computing. Vienna, Austria: R Foundation for statistical computing, 2010. http://www.R-project.org

18. Harrington DP, Flemming TR. A class of rank test procedures for censored survival data. Biometrica 1982;69:553-66.

19. World Health Organization. Integrated management of childhood illness. Geneva, Switzerland: WHO, 1999.
20. Young Infants Clinical Signs Study Group. Clinical signs that predict severe illness in children under age 2 months: a multicentre study. Lancet 2008;371:135-42.

21. Anderson P. Absence of evidence is not evidence of absence: we need to report uncertain results and do it clearly. UK Cochrane Centre. Br Med J 2004;328:476-7.

22. Gogia S, Sachdev HS. Home visits by community health workers to prevent neonatal deaths in developing countries: a systematic review. Bull World Health Organ 2010;88: 658-666B.

23. Kirkwood BK, Manu A, Asbroek AHA, et al. Effect of the Newhints home-visits intervention on neonatal mortality rate and care practices in Ghana: a cluster randomised controlled trial. Lancet 2013;381:2184-92.

24. Dubey AP, Rajeshwari K, Chakravarty A, et al. Use of VSL[sharp]3 in the treatment of rotavirus diarrhea in children: preliminary results. $J$ Clin Gastroenterol 2008;42(Suppl 3 Pt 1):S126-9.

25. Pammi M, Abrams SA. Oral lactoferrin for the treatment of sepsis and necrotizing enterocolitis in neonates. Cochrane Database Syst Rev 2011;(1):CD007138.

26. Gregory KE, De Forge CE, Natale KM, et al. Necrotizing enterocolitis in the premature infant. Adv Neonatal Care 2011;11: 155-66.

27. Ohlsson A, Lacy JB. Intravenous immunoglobulin for preventing infection in preterm and/or low-birth-weight infants. Cochrane Database Syst Rev 2013;(1):CD000361.

28. Rekha C, Whelan RM, Reddy P, et al. Evaluation of adjustment methods used to determine prevalence of low birth-weight babies at a rural hospital in Andhra Pradesh, India. Indian J Public Health 2013;57:177-80. 